\section{Revista Chilena de Pediatría}

wWw.revistachilenadepediatria.cl

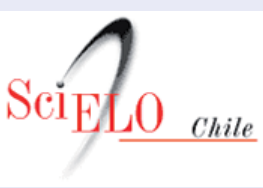

www.scielo.cl

\title{
Tabaquismo en el adolescente
}

\section{Adolescents and smoking}

\author{
Ana María Herrera ${ }^{\mathrm{a}, \mathrm{b}, \mathrm{c}}$, María Paz Corvalán ${ }^{\mathrm{d}, \mathrm{e}}$
}

aDepartamento Enfermedades Respiratorias Pediátricas Clínica Santa María

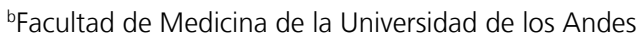

'Coordinadora Comisión Asma Infantil, Sociedad Chilena de Enfermedades Respiratorias

dPrograma de Tabaquismo Centro Médico Fundación del Banco Estado

eCoordinadora Comisión Tabaco, Sociedad Chilena de Enfermedades Respiratorias

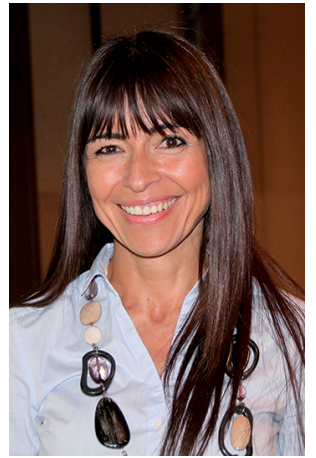

Ana María Herrera

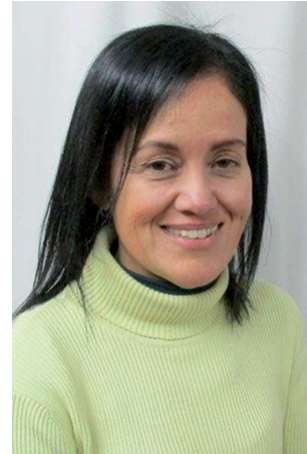

María Paz Corvalán
Esta adicción es un importante problema de salud pública a nivel mundial. De acuerdo a la Organización Mundial de la Salud cerca de 1 billón de hombres y 250 millones de mujeres fuman ${ }^{1}$. Se estima que 82.000 a 99.000 jóvenes al día se inician en la adicción al tabaco, muchos de los cuáles son menores de 10 años ${ }^{1}$. En relación a la prevalencia de tabaquismo en Chile, la tendencia de la serie de estudios del SENDA ${ }^{2}$ en población escolar chilena, muestra una sistemática reducción de la prevalencia de consumo en el último mes (desde 51,6\% a 40\% entre los años 2001-2015) y por último la Encuesta Mundial de Tabaquismo en Jóvenes, EMTJ3 Chile 2016, lanzada recientemente, evidencia un cambio significativo (desde $34,2 \%$ a $17,2 \%$ entre los años 2008 y 2016) en prevalencia de fumadores actuales de cigarrillos en la población de 13 a 15 años de la Región Metropolitana (fumaron cigarrillo en algún momento de los últimos 30 días), también hubo disminución significativa en el consumo en el resto de las regiones evaluadas en este estudio: Arica y Parinacota, Tarapacá, Valparaíso y Bío-Bío. Este es el resultado de la abogacía por políticas públicas realizada durante más de 20 años por un grupo de profesionales chilenos, muchos de ellos médicos. Si bien existen cambios importantes, aun es necesario bajar el consumo.

En la población pediátrica nos enfrentamos a dos serios problemas, uno es el inicio de la adicción en el adolescente y el otro es la exposición de los niños más pequeños al humo de segunda mano. La edad de inicio de la adicción al tabaco en Chile es a los 13,6 años en ambos sexos ${ }^{4}$.

Casi el 100\% del tabaquismo se inicia en la niñez y adolescencia, por lo que nuestra labor como pediatras es evitar que esto suceda. Los esfuerzos del control del tabaco deben estar puestos: en políticas públicas (aumento del impuesto al tabaco, campañas publicitarias masivas, ambientes cerrados libres de humo, restricción de la publicidad), la pesquisa precoz y tratamiento oportuno del adolescente que ya se ha iniciado en el consumo de tabaco. Al respecto creemos que es de vital importancia que las distintas sociedades científicas, dentro de las cuales la Sociedad Chilena de Pediatría cumple un rol central, presten su apoyo a todas estas iniciativas, especialmente en la terapia de cesación del tabaco realizada a través de la consejería breve o $\mathbf{A B C}$ $\mathrm{D}$, (se puede a realizar en 1 a 3 minutos) y registrar en la ficha clínica ${ }^{5}$. A: significa averiguar si el paciente fuma o no (debe hacerse en todo consultante independiente del motivo de consulta). B: implica dar un breve consejo firme, personalizado y repetido cada vez que un fumador consulte (en general los pacientes requieren varios intentos para dejar de fumar definitivamente), hablar del mal olor de la ropa, del color amarillo de la piel, dientes y uñas, la producción de arrugas, la sequedad del pelo, infertilidad e impotencia y C: cesación del tabaquismo que quiere decir (I) Referir a consejería te- 
lefónica (MINSAL Salud Responde, 6003607777, este número está escrito en los envases de cigarrillos), a un sitio $w^{2} b^{6}$, o a un centro que tenga implementado tratamiento de cesación del tabaquismo, o (II) Tratar si el paciente quiere dejar de fumar de aquí a un mes más.

Si fuma más de 10 cigarrillos al día o si fuma su primer cigarrillo durante la primera media hora desde que se levanta requiere terapia farmacológica, si existe patología psiquiátrica, debe estar estable por tres meses lo que permite el uso seguro de chicles de nicotina. Establecer la fecha para dejar de fumar o "Día D", (primer día sin fumar), que debe ser máximo hasta un mes más desde la primera consulta, e indicar al paciente que empiece a postergar $10 \mathrm{~min}$ cada cigarrillo y que haga, algo que lo distraiga (cambiar de actividad, consumiendo, chicles, líquidos, frutas, verduras. Si pasados los 10 min el paciente sigue con ganas de fumar puede hacerlo, recordar al paciente que no se le pide dejar de fumar, solo postergar. Prescribir medicamentos, si esto beneficia al paciente, en este caso chicles de nicotina que se usan de $2 \mathrm{mg}, 5$ a 8 al día SOS para disminuir el craving o deseo urgente de fumar. Vigilar que los pacientes usen el número adecuado de chicles, ya que tienden a usar menos, 1 cigarrillo es igual a $1 \mathrm{mg}$ de nicotina. Seguimiento a la semana para ver tolerancia al fármaco, (ya sea personal o telefónico) y controlar una vez a la semana en 3 ocasiones más ${ }^{5}$, (los mayores riesgos de recaída son durante el primer mes) ${ }^{5}$. Por último, $\mathbf{D}$ implica derivar siempre, si es que no dispone de tiempo para realizar el ABC. Más, detalles consignados en las Guías Clínicas de Tratamiento del Tabaquismo, Chile 2017 (http://dx.doi.org/10.4067/s071773482017000300167).

Tratar se hace urgente ya que los niños y adolescentes representan potenciales nuevos consumidores para la industria por lo que sus estrategias de mercadeo están directamente dirigidas hacia ellos.

A pesar de que en el último tiempo, gracias a la legislación, se ha restringido la publicidad casi en su to- talidad, la industria ha optado por otra estrategia para atraer a los jóvenes, que es el uso de aditivos químicos como el mentol, en los cigarrillos. Estos mejoran el sabor y hacen que el humo del cigarrillo se sienta más suave, reduciendo la irritación de la garganta y favoreciendo que al fumador novato le sea más fácil iniciarse en el tabaquismo ${ }^{6}$. Desgraciadamente la ley de tabaco en nuestro país no contempla la prohibición del uso de estos aditivos, por lo cual se hace urgente la aprobación de la nueva ley de tabaco que se encuentra en el parlamento.

Otro problema que debemos enfrentar como pediatras es la exposición de nuestros niños al humo de segunda mano ${ }^{7}$. Al respecto hay poca conciencia de los padres en relación a las serias consecuencias que esto tiene para la salud respiratoria de sus hijos, existe un estudio realizado en Inglaterra en 2003 el cual evidencia que cerca de 40 a $60 \%$ de los niños estaban expuestos al humo de segunda mano en sus casas. ${ }^{8}$. $\mathrm{i}$ bien la EMTJ, Chile 2016 evidencia disminución en la exposición al humo de tabaco ajeno en el hogar estadísticamente significativa en las regiones de Tarapacá y Metropolitana (de 46,8 a 38,3 y de 57,6 a 38,6 respectivamente) entre los años 2008 y 2016, la exposición es todavía extremadamante alta ${ }^{3}$.

Es frecuente que al preguntar acerca del tabaquismo en los padres éstos lo nieguen o lo minimicen. Dada la alta prevalencia de este problema nuestra labor como pediatras es indagar acerca de esta exposición en cada consulta. En caso de pesquisarse tabaquismo en los padres nuestra obligación es derivar a los padres a cesación del tabaco que puede ser telefónica (6003607777, Salud Responde MINSAL, este número aparece en las cajetillas de cigarrillos) o a algún centro que preste terapia de cesación del tabaquismo.

Nuestra labor es clave en la prevención y protección de la salud respiratoria de nuestros niños, solo así podremos tener futuros adultos libres de adicción al tabaco.

\section{Referencias}

1. Boletín de la Organización Mundial de la Salud. http://www.who.int/bulletin/ volumes/88/1/09-069583/en/ (Accedido en mayo 2017).

2. Servicio Nacional para la Prevención y Rehabilitación del Consumo de Drogas y Alcohol (SENDA). Décimo primer estudio nacional de drogas en población escolar (2015).Observatorio Chileno de Drogas. Disponible en: http://www.senda. gob.cl/media/estudios/PE/Informe\%20 Nacional\%20Final\%20ENPE\%202015.pdf (Consultado el 7/05/2017).
3. Encuesta Mundial de Tabaquismo en Jóvenes 2016, disponible en http://web. minsal.cl/wp-content/uploads/2017/10/ EMTJ2016_17_10_2017_VF.pdf

4. WHO Report on the Global Tobacco Epidemic, 2009. Appendix IX Global Youth TobaccoSurvey.http://www.who. int/tobacco/mpower/2009/appendix_ix/ en/index.html (Accedido en diciembre 2016)

5. McRobbie H, Bullen C, Glover M, Whittaker R, Wallace-Bell M, Fraser T. New Zealand smoking cessation guidelines. $\mathrm{N} Z \mathrm{Z}$ Med J. 2008;121(1276):57-70
6. Preventing tobacco use among youth and young adults. A report of the surgeon general.https://www.surgeongeneral. gov/library/reports/preventing-youthtobacco-use/exec-summary.pdf (Accedido en diciembre 2016).

7. Tutka P, Wielosz M and Zatonski W. Exposure to environmental tobbaco smoke and children health. Int J Occup Med Environ Health 2002; 15(4):325-35.

8. Rushton L, Courage C and Green E. Estimation of the impacto n children's health of environmental tobacco smoke in England and Wales. JRSH 2003; 123(3): 175-80. 